(63.4\%) men who have sex with men only; 34 (8.2\%) men who have sex with both men and women. Among the women: 74 (73.3\%) African-American; 18 (17.8\%) White; 6 (5.9\%) women who have sex with women only; 8 (7.9\%) women who have sex with women and men. Among men, the prevalence of CT was $10.7 \%(2.7 \%$ throat, $5.8 \%$ rectal and $3.4 \%$ urine); for GC $8.5 \%$ (6.5\% throat, $3.4 \%$ rectal and $1.2 \%$ urine). Among women, the prevalence of CT $12.9 \%$ (4.9\% throat, $8.9 \%$ rectal and $8.9 \%$ urine); GC 3.0\% (1.0\% throat, $3.0 \%$ rectal and $1.0 \%$ urine). $95.9 \%$ of the individuals reported high acceptance of self-testing with $97.6 \%$ willing to do repeat testing and $96.7 \%$ to recommend self-testing to someone else.

Conclusion HIV-negative, asymptomatic adults testing for HIV are infected with other STIs. Self-testing for CT and GC was implemented successfully among men and women. Self-testing can mitigate HIV infection by increasing detection and treatment of STIs amongst those seeking HIV testing only.

\section{P3.183 BORDERNETWORK: BRIDGING GAPS IN BIOBEHAVIOURAL STI SURVEILLANCE IN EUROPE}

doi:10.1136/sextrans-2013-051184.0640

${ }^{1} \mathbf{K}$ Haar, ${ }^{2 T}$ Arsova-Netzelmann, ${ }^{1} \mathrm{~V}$ Bremer, ${ }^{3} \mathrm{E}$ Vinzelj-Horvath, ${ }^{4} \mathrm{P}$ Komericki, ${ }^{5} \mathrm{~V}$ Benea, ${ }^{6} \mathrm{G}$ Tiplica, ${ }^{7} \mathrm{E}$ Petrova, ${ }^{8} \mathrm{D}$ Stanekova, ${ }^{2} \mathrm{E}$ Steffan. 'Department of Infectious Disease Epidemiology, Robert Koch-Institute, Berlin, Germany; ${ }^{2}$ SPI Forschung GmbH, Berlin, Germany; ${ }^{3}$ Magistratsabteilung 15 - Gesundheitsdienst der Stadt Wien Ambulatorium zur Diagnose und Behandlung sexuell übertragbarer Krankheiten, Vienna, Austria; ${ }^{4}$ Klinische Abteilung für Umweltdermatologie u. Venerologie, Universität Graz, Graz, Austria; ${ }^{5}$ Dermato-Venerological Unit, Clinical Hospital "Prof.Dr. Scarlat Longhin", Bucharest, Romania; ${ }^{6}$ Clinica Dermatologie 2, Spitalul Clinic Colentina, UMF "Carol Davila", Bucharest, Romania; 'Department of Dermatology and Venereology, Medical University, Sofia, Bulgaria; ${ }^{8}$ Slovak Medical University, National Reference HIVIAIDS Centre, Bratislava, Slovakia

Background Within the BORDERNETwork-project a biobehavioural sentinel surveillance was established in Austria, Bulgaria, Romania and Slovakia in 2010-12. The objectives were to record lab-confirmed STI (chlamydia, gonorrhoea, syphilis, HIV) in clinical settings and merge them with demographic and behavioural data to assess migration aspects, vulnerable groups and risk factors and recognise necessity for targeted interventions.

Methods On a monthly base, physicians reported aggregated STI testing data. Individual data was provided for each positive patient including demographics, re- and co-infections and assumed risk behaviour. Via patient questionnaires, information on socio-demographics, way of transmission and sexual behaviour was collected. All questionnaires were sent via regional to coordinating partners for merging and analysis.

Results Overall, 467797 tests were performed in 45 sentinel sites (Austria 13, Slovakia 14, Romania 13, Bulgaria 5). The countries varied in the number of STI tests (range: 6071 -298645), positivity rate (range: $2-13 \%$ ), patient characteristics and sexual behaviour. $75 \%$ of all women with an STI in Austria were sexworkers, compared to 5\% and $8 \%$ in Bulgaria and Romania. 34\% of all men with an STI in Slovakia had sex with men, compared to $4 \%$ in Romania. STI patients, especially women had a migration background in $79 \%$ in Austria, but less than $7 \%$ in the other three countries. Casual partners were the presumed cause of infection in the majority of MSM in all countries, whereas condom use in the last 6 months with these partners varied significantly between the 4 countries.

Conclusions Although not representative, sentinel surveillance gathers useful information on groups most at risk and can be compared between countries when using the same instruments. Legal and social issues can hinder disclosure of sexual preferences and practises and hamper targeted prevention. Enhancement of condom use with casual partners in MSM seems to be crucial, particularly in the East.

\section{P3.184* SEXUALLY TRANSMITTED INFECTIONS AND PREGNANCY OUTCOMES, FLORIDA 2008-2010}

doi:10.1136/sextrans-2013-051184.0641

K. Schmitt, D. R. Thompson. Florida State University, Tallahassee, FL, United States.

Background A prior population based study found the odds of low birth weight for women with inadequate weight and history of Chlamydia trachomatis $(\mathrm{Ct})$ infection during the pregnancy was nearly twice that of women without Ct infection (AOR 1.98, $\mathrm{p}<0.02)$. A stronger association was observed with pre-term low birth weight (AOR 2.34, p < 0.01). Subsequently, 2005-2006 ratified state laws and regulations required $\mathrm{Ct}$ screening during pregnancy. Objectives To examine associations between $\mathrm{Ct}$ infections during pregnancy and birth outcomes among women who gave birth in Florida after implementation of mandated Ct testing.

Methods Logistic Regression analysis of Florida birth records $(548,407)$ linked to records for women who tested positive for $\mathrm{Ct}$ $(12,334)$, were treated and gave birth from 01/01/2008 to 12/31/2010.

Results Among 18-35 years old, with singleton births, $2.7 \%$ were reported with chlamydia during pregnancy and $6.5 \%$ experienced low birth weight (LBW) outcome, less than 2500 grammes. After controlling for smoking, prenatal care, race, education, marital status, inadequate weight gain and nine other covariates, Ct infection treatment in gestation weeks 17 through 28 was associated with increased odds of delivering a moderately LBW infant (1500 to 2499 grammes) (AOR 1.29, 95\% CI 1.12 to 1.49). No association was found for very LBW infants ( $<1500$ grammes) or for Ct infections treated before 17 weeks gestation. The increased odds for moderately LBW were observed for preterm ( $<37$ weeks gestation) moderately LBW infants (AOR $1.28,95 \%$ CI 1.07 to 1.53$)$ and term ( $\geq 37$ weeks gestation) moderately LBW infants (AOR 1.27, 95\% CI 1.03 to 1.57$)$.

Conclusions Proactive public health policies for chlamydia screening during pregnancy and intensified treatment timeliness may have contributed to improved pregnancy outcomes, but Ct infection continues to be associated with increased odds of moderately LBW. Prospective examination of treatment dosage and timeliness may prove informative.

\section{P3.185 CHLAMYDIA TRACHOMATIS IN THE GENITAL TRACT OF HIV-INFECTED WOMEN, PREGNANT AND NON-PREGNANT, FOLLOWED IN A REFERENCE CENTRE IN BAHIA, NORTHEAST BRAZIL}

doi:10.1136/sextrans-2013-051184.0642

'A G A Travassos, "I Nóbrega, ${ }^{2} E$ Netto, ${ }^{2} \mathrm{M}$ Timbó, 'K Adami, 'S Fernandes, ${ }^{1} \mathrm{P}$ Silva, ${ }^{1} \mathrm{M}$ Pires, ${ }^{2} \mathrm{C}$ Queiroz, ${ }^{2} \mathrm{C}$ Brites. ${ }^{1}$ Centro Estadual Especializado em Diagnóstico, Assistência e Pesquisa (CEDAP-SESAB), Salvador, Brazil; ' Universidade Federal da Bahia, Salvador, Brazil

Background Genital tract infection by Chlamydia trachomatis(CT) is associated with complications such as pelvic pain, infertility and ectopic pregnancy, besides increased risk of HIV sexual transmission. Its occurrence during gestation worsens perinatal morbidity and mortality. In HIV-infected pregnant women also increases the risk of HIV mother-to-child transmission despite the use of HAART. In Brazil, there are few data on the prevalence of HIV/CT co-infection and associated risk factors.

Methods Cross-sectional study of HIV-infected women attending in a reference outpatient clinic for gynaecology and prenatal care between October 2010 and September 2011 was conducted, evaluating laboratory and clinical data. The search of $C T$ was performed using Hybrid Capture II collected from endocervix, and clinical data were obtained from standard questionnaire and survey data in medical records. 
Results 112 HIV-infected women were enrolled, of whom 52 nonpregnant and 60 pregnant. In this population, mean age was 32.3 years ( $\mathrm{SD}=8.2), 62.5 \%$ had a previous history of sexually transmitted diseases, $46.4 \%$ began sex life with 15 years or less, $33.1 \%$ reported having less than 3 sexual partners throughout life, $45.8 \%$ had undetectable HIV viral load. We found a prevalence of $5.4 \%$ of Chlamydia trachomatis infection in HIV-infected women followed. There was an association of CT with the presence of pregnancy $(10.0 \%$ versus $0.0 \% ; p=0.019)$, HIV viral load $>10,000$ copies $(p<0.001)$ and the mean time of HIV diagnosis $(21.0$ versus 69.2 months; $p=0.032$ ). We found no association with other risk factors studied (ethnicity, marital status, education, use of alcohol and drugs, CD4+ T Lymphocyte count).

Conclusion Early access to diagnosis and treatment of infection by HIV and Chlamydia trachomatis is an important preventive action. In pregnant women infected with HIV, the prevalence of Chlamydia appears to be greater and this is a period where treatment can improve maternal and neonatal outcome.

\section{P3.186 SUBSTANTIAL NATURAL CLEARANCE OF GENITAL AND EXTRAGENITAL CHLAMYDIA TRACHOMATIS AND NEISSERIA GONORRHOEAE IN STD CLINIC ATTENDEES}

doi:10.1136/sextrans-2013-051184.0643

. 2 A F S van Liere, ${ }^{1,2}$ N H T M Dukers-Muijrers, ${ }^{2}$ P F G Wolffs, ${ }^{1,2} \mathrm{C}$ J P A Hoebe. 'Department of Sexual Health, Infectious Diseases and Environmental Health, Public Health Service South Limburg, Geleen, The Netherlands; '2Department of Medical Microbiology, Maastricht University Medical Center, Maastricht, The Netherlands

Background Little is known on the natural history of extragenital Chlamydia trachomatis $(\mathrm{Ct})$ and Neisseria gonorrhoeae $(\mathrm{Ng})$. More insight in the natural history of extragenital $\mathrm{Ct}$ and $\mathrm{Ng}$ could influence standard operating procedures in screening facilities such as STD clinics. We evaluated proportions of natural clearance of $\mathrm{Ct}$ and $\mathrm{Ng}$ in genital and extragenital samples.

Methods We included self collected $\mathrm{Ct}$ and/or $\mathrm{Ng}$ positive genital (urine and cervicovaginal) and extragenital (anorectal and oropharyngeal) samples from STD clinic attendees [April 2011-December 2012]. Data on demographics and sexual behaviour were collected in an electronic patient file at initial testing. At follow-up for treatment, subjects were retested prior to treatment at the same anatomic site(s) as initial testing and provided an additional questionnaire on sexual behaviour since initial testing. Natural clearance rates of $\mathrm{Ct}$ and $\mathrm{Ng}$ were compared between anatomic sites. Data on the first 238 individuals (299 samples) are presented, enrollment is ongoing.

Results In total, $38 \%$ of the participants were male and the median age was 27 years. The median interval between initial and follow-up sample was 11 days. Natural clearance of Ct was 10.5\% (22/210) for genital samples (36\% urine, 64\% vaginal) and $25.9 \%$ (7/27) for extragenital samples (57\% anorectal, $43 \%$ oropharyngeal) $(\mathrm{P}=0.49)$. For $\mathrm{Ng}$ this was $22.2 \%$ (2/9) for genital samples (0\% urine, $100 \%$ vaginal) and $18.8 \%(3 / 16)$ for extragenital samples (33\% anorectal, $67 \%$ oropharyngeal) $(\mathrm{P}=0.84)$. Overall, natural clearance of $\mathrm{Ct}$ was $12.2 \%(29 / 237)$ and $\mathrm{Ng}$ was $20.0 \%(5 / 25)(\mathrm{P}=0.14)$. Age $<25$ years $(\mathrm{P}<0.01)$ and female sex $(\mathrm{P}=0.03)$ were associated with overall $\mathrm{Ct}$ clearance. Median interval was not associated with $\mathrm{Ct} / \mathrm{Ng}$ clearance ( $\mathrm{P}=0.13$ and $\mathrm{P}=0.11$ respectively).

Discussion Natural clearance of $\mathrm{Ct}$ and $\mathrm{Ng}$ was substantial in both genital and extragenital samples. Further analysis on associated determinants as well as bacterial load determinations will provide more insight into these results.

\section{P3.187 SEXUALLY TRANSMITTED INFECTIONS AMONG A COHORT OF HORMONAL CONTRACEPTIVE USERS IN IBADAN, NIGERIA}

doi:10.1136/sextrans-2013-051184.0644
S A Fayemiwo, A A Fatiregun, R A Bakare. College of Medicine, University of Ibadan, Ibadan., Ibadan, Nigeria

Background Most women who acquire HIV and other sexually transmitted infections (STIs) are in their child bearing years and are current or potential users of contraceptive methods. The study was undertaken to provide information on the association between the hormonal contraceptive methods and STIs among women attending Family Planning clinics, University College Hospital,Ibadan,Nigeria.

Methods It was a cross-sectional study in a population of women using hormonal contraceptive methods attending Family Planning clinics. Detailed medical history, Endocervical and high vaginal swabs were collected from the women to establish diagnosis after clinical examination and informed consent. Aliquots of sera from venous blood samples of the women were tested for antibodies to HIV-1/2 and RPR. Data was analysed using SPSS for widows' version 15.0 .

Results There were 102 women using hormonal contraceptive methods who participated in the study with mean age of 31.92 years $(\mathrm{SD}=8.33$, range $=16-55)$. The mean age of sexual debut of participants was 19.5 years. The most common STI diagnosed was Vaginal candidiasis $(22.5 \%)$ while others were bacterial vaginosis (21.6\%), HIV (11.8\%), Trichomoniasis (11.8\%), Chlamydia cervicitis $(8.8 \%)$, syphilis $(5.9 \%)$, genital warts $(6.9 \%)$ and gonorrhoea $(2.9 \%)$.Younger age of sexual debut influenced the decision of selecting various forms of hormonal contraceptives especially the emergence of oral contraceptive forms ( $\mathrm{P}=0.043$. Majority of the women on hormonal contraceptives had multiple sexual partners. There were significant associations between utilisation of hormonal contraceptive methods and transmission of trichomoniasis $(\mathrm{P}=0.019$, $4.2(1.0-13.2)$.

Conclusions Women seeking contraception to prevent unintended pregnancy are as much in need of education about prevention of STIs. The study found that younger age, numbers sexual partners, and use of hormonal contraceptives could increase the risk of acquiring trichomonads infection.

\section{P3.188 CYTOMEGALOVIRUS AND HIV CO-INFECTION AMONG PATIENTS ACCESSING CARE IN A TERTIARY CARE CENTRE IN NIGERIA}

doi:10.1136/sextrans-2013-051184.0645

'A Fowotade, ${ }^{2} \mathrm{O}$ Agbede, ${ }^{2} \mathrm{~A}$ Salami, , $\mathrm{A}$ Fayemiwo, ${ }^{1} \mathrm{~A}$ Efunshile. 'University of Ibadan, Ibadan, Nigeria; ' University of Ilorin, Ilorin, Nigeria

Background Cytomegalovirus (CMV) is one of the commonest viral opportunistic infections in persons with Acquired Immune Deficiency Syndrome (AIDS). In AIDS patients, progressive loss of immune function, and in particular, loss of cell mediated immunity, permits CMV reactivation and replication. This study aimed to evaluate the prevalence of seropositivity for CMV IgG and IgM among HIV sero-positive patients in Ilorin, Nigeria

Methodology Sera obtained from 180 HIV sero-positive individuals and 180 HIV sero-negative blood donors participants were assayed for CMV IgG/IgM using Enzyme Linked Immunosorbent Assay (ELISA), The CD4 cell counts were also done. A semi-structured questionnaire was used to obtain information on the risk factors for CMV/HIV co-infection.

Result A total of $169(93.9 \%)$ of the HIV sero-positve were CMV IgG positive while, 174(96.7\%) of the control were also CMV IgG positive. Among the HIV sero-positive, 20 (11.1\%) were CMV IgM antibody positive, while $4(2.2 \%)$ of the HIV sero-negative control group were CMV IgM positive. Age of Participants $(p=0.000)$, number of sexual partners $(p=0.000)$ and CD4 cell counts $(p=0.000)$ were significantly related to CMV IgM sero-positivity. However the use of HAART $(p=0.777)$, history of blood transfusion $(p=0.837)$ 